\title{
Estudio toxicológico con dosis subaguda de Proustia Berberidifolia (tasa) en ratas de laboratorio
}

\author{
Toxicological Study with Subacute Dose of Proustia \\ Berberidifolia (tasa) in laboratory rats
}

1 Ricardo Ortiz Faucheux

\begin{abstract}
RESUMEN:
Se diseñó un estudio prospectivo, longitudinal, de intervención. comparativo-explicativo para probar la hipótesis: que la administración $(300 \mathrm{mg} / \mathrm{kg} \mathrm{pc})$ en forma prolongada del extracto acuoso de Proustia berberidifolia (tasa), a la cual la creencia popular le atribuye propiedades terapéuticas ,produce modificaciones negativas en los parámetros hioquimicos sanguineos de rattus norvegicus de la variedad ways, los cuales se midieron al inicio, catorce y veintiocho días. Objetivo: Fue establecer el efecto del extracto sobre la bioquímica sanguinea. Material y métodos: El extracto se preparó a base de planta seca y estabilizada. Se administró mediante sonda gástrica. Se utilizaron cinco animales de cada sexo con sus respectivos controles haciendo un total de veinte. Los grupos control en lugar de extracto recibieron agua. Resultados: bilirmbinas, transaminasas y colesterol lotal modificadas. Conclusiones: Contradictoriamente las modificaciones observadas son positivas y están relacionados con efectos depurador, desintoxicador hepatoprotector e hipolipemiante.
\end{abstract}

Palabras clave: Depurador: desintoxicador: hepatoprotector:

\section{ABSTRACT:}

It was designed a prospective, longitudinal, intervention, explanatory-comparative study to tas the hypothesis: that the administration $(300 \mathrm{mg} / \mathrm{kg}$ bw) in a prolonged way of berberidifolia Prouslia aqueous extract (tasa), to which popular belief attributed therapeutical properties produces adverse changes in blood biochemical parameters of the variety rattus norvegicus Ways, which are measured at the beginning, fourteen and twenty-eight days. Objective: to establish the effect of the extract on blood chemistry and methods The extract is prepared from dried plant and stabilized. It was administered by gavage. Five animals of each sex with their respective controls for a toral of twenty were used. Control groups received water instead of the extract. Results: bilirubin, transaminases and total cholesterol modified Conclusions:Contradictorily to the observed changes, they are positive and are related to effects debugger, hepatoprotective detoxifying and lipid lowering.

Keywords: debugger, detoxifier, hepatoprotective.

' Doctor en Ciencias de la Salud. Mgr en fisiopatologia, Quimico Farmacéutico. Facultad de Ciencias de la Salud Universidad Nacional Jorge Basadre Grohmann 


\section{INTRODUCCIÓN}

Mucho se ha escrito acerca de la antigüedad del uso de las plantas medicinales como recurso terapéutico popular (Carretero, 1998). Como se sabe una planta está constituida por una variedad de compuestos quimicos de mayor o menor complejidad y son estos componentes los que interactúan con una gran diversidad de moléculas biológicas que pueden ser receptores de la membrana celular $y / 0$ citoplasmáticos, sistemas transportadores de iones, enzimas etc. Esta caracteristica determina que estos sistemas biológicos en equilibrio, por la presencia de sustancias complementarias (Rodriguez, 1998), no actúan por fuerzas inexplicables o desconocidas. muy por el contrario, está cada vez mas establecido que lo hacen por las mismas vías utilizadas por los medicamentos llamados convencionales y no pocas veces, a través de varias vias simultáneas, en lo que se denomina acción sinergistica.

De allí que existan antecedentes de interacciones entre medicamentos y plantas medicinales (Chan, 2000; Duclos. 2001 e lzzo, 2001), sin embargo, lo cierto es, que conforme avanzan las investigaciones se observan que unas plantas medicinales son realmente eficaces para ciertos tratamientos (Izzo, 200I y Luper, 1998). pero a la vez pueden producir reacciones adversas como dermatitis (Schatzle, 1998). hemorragia intracerebral (Matthews. 1998), anafilaxia (Myer, 1998), colestasis (Tomas, 1998) nefrotoxicidad (Conz, 1998), hepatitis (Gaviln, 1999), ginetomastia (Palop, 1999), sindrome de Fanconi (Krumme, 2001) como se describe en el sistema Español de Farmacovigilancia (F.E.D.R.A).

Por lo tanto de manera similar a los medicamentos alopáticos, se aconseja usar las plantas con cautela en ciertos grupos de personas, especialmente: niños (Tomassoni, 2001), personas de tercera edad (Parkman, 2001), así como en pacientes con patologias renales (Foote, 2001) y enfermos con cáncer (Oneschuk, 1999).

En la medida que las plantas medicinales cuenten con un respaldo científico (Price, 1982 y Bourgoignic 1981) avalados por ensayos farmacológicos y toxicológicos tanto in vitro como in vivo asi como por ensayos clínicos su uso será seguro.

Se debe considerar también, que a pesar del aumento de las investigaciones y estudios científicos en este campo, todavia no se conocen los principios activos responsables de las cualidades de muchas de ellas (Myer, 1998); en consecuencia habrá que cuestionarse la seguridad de la utilización de plantas de composición incierta ( Woo, 1979) y más aún si su consumo va a ser por tiempo prolongado. Por eso que toda planta a la que la población le atribuya bondades terapéuticas antes de administrarse a los seres humanos deben ser sometidas a ensayos rigurosos. Nuestro trabajo de investigación sobre la Proustia berberidifolia (tasa) aporta información cientifica importante sobre los efectos benéficos a nivel hepático que tienen repercusión en el organismo en general.
Al final, la complementación con más estudios científicos sobre esta planta permitirá producir una nueva categoria de fármaco (fitofármaco) donde se fusiona el conocimiento ancestral etnobotánico y etnomédico; sumados a los modernos conocimientos en farmacologia y tecnologia farmacéutica obteniéndose así un producto a base de un extracto natural estandarizado con una forma farmacéutica adecuada que no guarda diferencia en su aspecto y calidad con los medicamentos alopáticos tradicionales y estará en nuestros anaqueles como una alternativa más para la prescripción cuando de enfermedades o de proteger el hígado se trate.

\section{MATERIALESY MÉTODOS.}

El trabajo de investigación desarrollado fue analítico experimental (prospectivo, longitudinal, de intervención, comparativo-explicativo) donde se usaron dos poblaciones y se procedió considerando la Tabla $\mathrm{N}^{\circ}$ 1. El tratamiento estadístico fué inferencial. Anova factorial de tres y dos factores con efectos fijos. multivariable multigrupos aleatorizado, cada unidad de análisis (rata) bajo un solo tratamiento con un número de replicas 18 UUA.

El método utilizado fue el de administración oral continuada (28 dias) y el material biológico estuvo constituido por 20 ratas albinas (Rutus norvegicus 10 ratas hembras, 10 ratas machos ) jóvenes y sanas de seis meses, similar peso que no sobrepasaron $+-20 \%$ del valor medio pertinente Las hembras fueron nulíparas y nográvidas.

Se mantuvo constantes las condiciones de temperatura. humedad, iluminacion, oscuridad natural y alimentación durante los dias que duro la investigación.

Los animales antes de entrar al tratamiento fueron preparados, la via de administración utilizada fue la oral. La forma farmacéutica fue extracto que fue preparado en el momento de las administraciones.

Los parámetros de bioquímica clinica estudiados en la muestra de sangre obtenida fueron realizados por espectrofotometría campo visible

\section{RESULTADOSY DISCUSIÓN}

Los resultados se presentan en la Tablas 2.3 y 4 , y en las Figuras 1, 2 y 3 se presenta el comportamiento de LDLcolesterol pos administración del extracto acuoso de proustia berberidifolia.(tasa). La administración del extracto acuoso de Proustia berberidifolia (tasa) a animales de experimentación afecta el funcionamiento hepático más no el renal. Se inidió la actividad de la mayoría de enzimas indicadoras de daño celular así como los parámetros bioquimicos indicadores de alteraciones metabólicas y en determinados casos se acompañó de pruebas destinadas a medir la capacidad funcional de órganos especificamente de los encargados de la metabolización o eliminación de un toxico o metabolito presente después de la ingesta de un xenobiótico. 
Tabla $N^{0} 1$ Matriz de Operacionalización

\begin{tabular}{|c|c|c|c|c|c|}
\hline Variables & Criterios & Procedimientos & Indicad. & Subindicadores & $\begin{array}{c}\text { Escala } \\
\text { de } \\
\text { Medición }\end{array}$ \\
\hline $\begin{array}{l}\text { Variable } \\
\text { Independiente } \\
\text { VI: } \\
\text { Dosis del } \\
\text { extracto acuoso } \\
\text { de Proustia } \\
\text { berberidifolia } \\
\text { (tasa) }\end{array}$ & $\begin{array}{l}\text { Dosis es la } \\
\text { concentración } \\
\text { de sustancia a } \\
\text { administrar }\end{array}$ & $\begin{array}{l}\text { Administración de } \\
\text { una dosis constante } \\
\text { de acuerdo al peso } \\
\text { corporal(pc) del } \\
\text { animal durante todo } \\
\text { el experimento }\end{array}$ & Dosis & $\begin{array}{l}\text { Grupos } \\
\text { Numero } \\
\text { Peso } \\
\text { Via de } \\
\text { administración } \\
\text { Volumen } \\
\text { administrado }\end{array}$ & $\begin{array}{l}\text { Numérica } \\
\text { continua }\end{array}$ \\
\hline $\begin{array}{l}\text { Variable } \\
\text { Dependiente } \\
\text { V2: } \\
\text { Efecto del } \\
\text { extracto acuoso } \\
\text { de } \\
\text { Proustiaberberi } \\
\text { difolia } \\
\text { (tasa) }\end{array}$ & $\begin{array}{l}\text { Cambios } \\
\text { cuantitativos en } \\
\text { los valores de } \\
\text { los parámetros } \\
\text { bioquímicos } \\
\text { antes de iniciar } \\
\text { el experimento, } \\
\text { a la mitad y al } \\
\text { final del mismo. }\end{array}$ & $\begin{array}{l}\text { medición de los } \\
\text { valores de los } \\
\text { parámetros } \\
\text { bioquimicos en } \\
\text { estado basal } \\
\text { medición de los } \\
\text { valores de los } \\
\text { parámetros } \\
\text { bioquímicos a los } \\
14 \text { dias después de } \\
\text { administrac. diarias } \\
\text { de extracto } \\
\text { medición de los } \\
\text { valores de los } \\
\text { parámetros } \\
\text { bioquimicos al } \\
\text { termino del estudio }\end{array}$ & $\begin{array}{c}\text { Pruebas } \\
\text { bioquími } \\
\text { cas }\end{array}$ & $\begin{array}{l}\text { Aspartatoaminotran } \\
\text { sferasa } \\
\text { (ASAT,GOT), } \\
\text { alaninoaminotransfe } \\
\text { rasa (ALAT,GPT), } \\
\text { bilirrubina total } \\
\text { directa e indirecta, } \\
\text { fosfatasa alcalina } \\
\text {,albúmina (Alb), } \\
\text { proteinas totales } \\
\text { (PT), glucosa } \\
\text { (Gluc), colesterol } \\
\text { total (c-T), c-HDL, } \\
\text { c-LDL } \\
\text {,triglicéridos (TG), } \\
\text { creatinina (Crea) y } \\
\text { ácido úrico } \\
\text { (AU).urea, } \\
\text { nitrógeno ureico } \\
\text { sanguíneo (BUN) }\end{array}$ & $\begin{array}{l}\text { Numérica } \\
\text { continua }\end{array}$ \\
\hline
\end{tabular}

pc (peso corporal)

Tabla N" 2 : Efecto Intersujetos Medición

\begin{tabular}{llccl}
\hline Fuente & Variables Dependientes & Significación & $\begin{array}{c}\text { Eta al Cuadrado } \\
\text { Parcial }\end{array}$ & Nivel De Efecto \\
\hline \multirow{3}{*}{ Tiempo de } & Brea & .593 & .008 & No afectado \\
Medición & Bilirrubina total & .033 & .120 & Bajo \\
(basal y 28 & Bilirrubina directa & .004 & .210 & Bajo \\
dias) & Bilirrubina indirecta & .324 & .027 & No afectado \\
& TGP & .000 & .294 & Bajo a medio \\
& TGO & .178 & .050 & No afectado \\
& Fosfatasa alcalina & .059 & .096 & No afectado \\
& Proteínas totales & .148 & .057 & No afectado \\
& Albumina & .660 & .005 & No afectado \\
& Amilasa & .074 & .086 & No afectado \\
& & .570 & .009 & No afectado \\
\hline
\end{tabular}


Tabla N" 3: Efecto Intersujetos Medición

\begin{tabular}{llccl}
\hline Fuente & Variables Dependientes & Significación & $\begin{array}{c}\text { Eta al Cuadrado } \\
\text { Parcial }\end{array}$ & Nivel de Efecto \\
\hline \multirow{4}{*}{ Tratamiento } & BUN & .672 & .005 & No afectado \\
$(300 \mathrm{mg} / \mathrm{Kg}$ & Bilirrubina total & .797 & .002 & No afectado \\
pc). & Bilirubina directa & .511 & .012 & No afectado \\
& Bilirrubina indirecta & .955 & .000 & No afectado \\
& TGP & .279 & .032 & No afectado \\
& TGO & .000 & .335 & Bajo a medio \\
& Fosfatasa alcalina & .347 & .025 & No afectado \\
& Proteinas totales & .072 & .087 & No afectado \\
& Albumina & .230 & .040 & No afectado \\
& Amilasa & .425 & .018 & No afectado \\
\cline { 2 - 4 } & & .563 & .009 & No afectado \\
\hline
\end{tabular}

Tabla $\mathrm{N}^{0} 4$ : Efectos Intersujetos Tratamiento

\begin{tabular}{clccl}
\hline Fuente & \multicolumn{1}{c}{$\begin{array}{c}\text { Variables } \\
\text { Dependientes }\end{array}$} & Significación & $\begin{array}{c}\text { Eta al Cuadrado } \\
\text { Parcial }\end{array}$ & Nivel de Efecto \\
\hline & Glucosa & 0.078 & 0.055 & Despreciable \\
& Creatinina & 0.052 & 0.067 & Despreciable \\
Tratamiento & Colesterol total & 0.002 & 0.156 & Bajo \\
$(300 \mathrm{mg} / \mathrm{Kg} \mathrm{pc})$. & HDL colesterol & 0.347 & 0.016 & Despreciable \\
& LDL colesterol & 0.078 & 0.055 & Despreciable \\
& Triglicéridos & 0.976 & 0.000 & Despreciable \\
& Acido ùrico & 0.700 & 0.003 & Despreciable \\
\hline
\end{tabular}

Uno de estos órganos de gran importancia por su nobleza y múltiples funciones es el higado. Se estima que lleva a cabo más de 500 actividades distintas, entre las que se encuentra la de desintoxicación, y cuando deja de funcionar se produce la muerte del organismo a las 10 horas (Price, 1982). Es un órgano con propiedades de regeneración total y tiene una capacidad de reserva considerable que le permite funcionar dentro de linites normales hasta que $80 \%$ de los hepatocitos se han destruido (Price, 1982). Las pruebas de evaluación de la función hepática se basan en la enorme gama de reacciones que alli ocurren, entre estas pruebas destacamos las diseñadas para medir la capacidad funcional (bilirrubina, carbohidratos, lípidos. proteinas y urea, entre otras), para medir la capacidad desintoxicadora (bilirrubina, amoniaco, alcohol, fármacos) para medir la capacidad excretora o secretora (ácidos biliares, colesterol, bilirrubina), para medir la capacidad almacenadora de: glucógeno, lipidos. aninoácidos y proteinas, hierro, cobre, vitaminas) para detectar daño celular del hepatocito (transaminasas) y de la vía biliar (bilirrubina directa, bilirrubina total y fosfatasa alcalina), y por último para medir la capacidad inmunológicas (fagocitosis, limpieza de bacterias entre otras sustancias extrañas, secreción de $\operatorname{IgA}$, defensas humorales), y también diagnosticar la etiología de la enfermedad, no siendo consideradas estas dos últimas pruebas en el presente trabajo por la extensión del mismo.

La bilirrubina producto final del catabolismo del grupo Hen (hemoglobina, citocromo $P_{45 n}$ ) se produce en el sistema reticulo endotelial y de alli pasa a la circulación siendo trasportada al higado unida a la albúmina (bilirrubina indirecta insoluble en agua). El hígado to conjuga con ácido glucorónico (bilirrubina directa soluble en agua) y lo excreta a la bilis. En el intestino por degradación bacteriana se transforma en urobilinógeno que pasa a las deposiciones. Alteraciones en la formación de bilirrubina, transporte o conjugación dan origen a un aumento de bilitrubina indirecta. en cambio una alteración de la excreción origina un aumento de la bilirrubina conjugada. La excreción inadecuada de bilirrubina conjugada puede ser producto de una obstrucción intra como extrahepática producida por una sustancia extraña, lo que no permiten hacer un - diagnóstico diferencial pero mientras más elevado sea el valor más probable es que la obstrucción sea extrahepática. En este estudio se demuestra que con la administración de la tasa varian indirectamente los niveles de bilirrubina total, bilirrubina indirecta por una óptima conjugación a nivel hepático. En concreto se ha mejorado la capacidad funcional del higado, en especial su capacidad excretora y desintoxicadora como lo 
demostraremos más adelante. no existiendo obstrucción intrahepática ni a nivel biliar lo que se corrobora con los niveles de fosfatasa alcalina encontrados que no presentaron variación alguna no comprometiendo otro órgano abdominal o estructura abdominal aledaña como puede ser el páncreas. Aunque se desconoce el mecanismo exacto que desencadena el ataque pancreático, con frecuencia $(75 \%)$ se relaciona con enfermedades de los conductos biliares o alcoholismo en caso de los seres humanos. El restante $25 \%$ de los casos, según Lott JA (24), se debe a otras causas, incluyendo las inducidas por fármacos e hiperlipemia.

Otras pruebas realizadas están vinculadas con la capacidad funcional de sintesis de proteinas totales. urea. metabolismo de lipidos y carbohidratos por parte del higado. Este sintetiza la mayoría de las proteínas plasmáticas con excepción de las gammaglobulinas que se sintetizan en el retículo endotelial La disminución de su capacidad funcional por alguna agresión conduce a un descenso del nivel de todas las proteinas plasmáticas, especialmente de la albúmina. Estas alteraciones no se reflejan inmediatamente ya que la vida media de las proteínas es mayor a 20 dias, por lo que esta prueba no es útil como test en el daño hepático agudo pero si en subagudo como es el caso nuestro, y mejor en el crónico. En las enfermedades hepáticas crónicas, especialmente la cirrosis, se produce una disminución de la albümina y una elevación de las globulinas (a expensas de las gammaglobulinas) y cuando se mide la proteina total este efecto se compensa dando niveles normales, por esto, la determinación de las fracciones proteicas tienen más valor diagnóstico. En la hepatitis la proteinemia total se encuentra dentro de los limites normales la albúmina discretamente descendida y la gama globulina algo aumentada. En las ictericias obstructivas hay alteraciones pequeñas que dependen de la duración del proceso. En el caso especifico nuestro los resultados (Tabla $\mathrm{N}^{\circ} 3$ ) demuestran que la administración de la tasa no afecta la producción de proteinas y albumina puesto que el comportamiento es similar en el grupo control y tratado de ambos sexos no existiendo por lo tanto un

\section{Medias marginales estimadas de Colesterol Total}

$$
\text { En macho hembra }=\text { macho }
$$

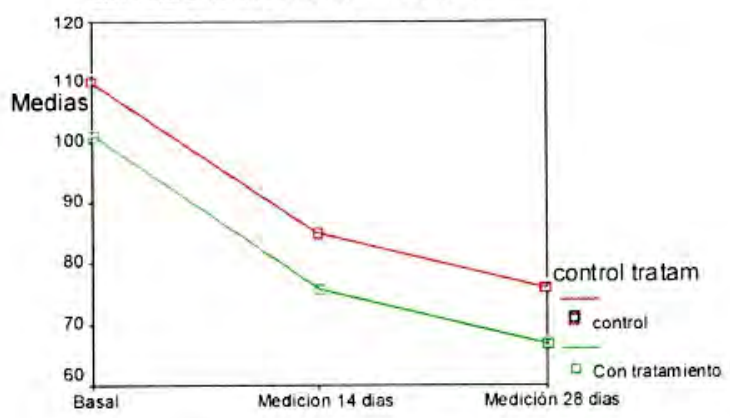

basal,14d,28d proceso subagudo maligno a nivel hepático.

En cuanto a la descomposición de proteinas y aminoácidos, es la urea el producto final la cual se sintetiza en el higado, se excreta por los riñones y la cuarta parte de esta se metaboliza en los intestinos para formar amoniaco y dioxido de carbono mediante la actividad de la flora bacteriana normal (Woo y Canon. 1979). Posteriormente este amoniaco se reabsorbe a través del sistema portal y se convierte de nuevo en urea en el higado. El aumento de niveles de amonio sanguineo indicará una disminución en la síntesis ureica por daño hepático. Los niveles de urea encontrados en nuestro trabajo indican un ascenso de esta sustancia especialmente en el grupo de hembras tanto del grupo tratado como del control, hecho que no se debe al efecto del extracto de la planta en estudio sino a otros factores excluido el factor sexo to que indicaría buena producción de urea a nivel hepático, y probablemente una disminución de la excreción a nivel renal en comparación con la de los machos.

De los resultados obtenidos a nivel metabólico podemos afirmar que a la dosis utilizada del extracto de Proustia berberidifolia el único parámetro bioquímico que es afectado directamente por el factor tratamiento es el colesterol total, cuyas fracciones (HDL y LDLcolesterol) también sufren variación pero por motivos variados. Del estudio se desprende (figuras $N^{0} 1,2$ y 3 ) que a medida que baja el nivel de colesterol total, que es significativo a partir de los catorce dias, la fracción que disminuye es el c-LDL mientras que el c-HDL presenta efecto inverso. Estos dos últimos efectos no atribuidos directamente al tratamiento (Tabla $\mathrm{N}^{\circ} 4$ ) nos inducen a pensar que la planta en estudio actuaria optimizando el funcionamiento del hígado (proceso de metabolización $y$ excreción) en especial la capacidad excretora lo que confirmaria lo dicho por Patsch W (Patsch, 1989) cuando se refiere a que el higado es capaz de excretar varios gramos de colesterol del organismo a través de las heces. En cuanto a los triglicéridos (TG) este parámetro no fue afectado por el factor tratamiento por lo que sus

\section{Medias marginales estimadas de Colesterol Total}

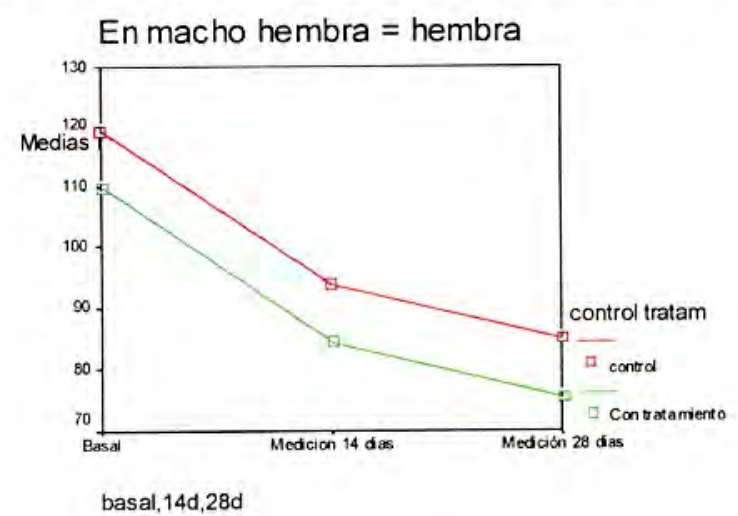

Figura N"01 Comportamiento de la variable DE L dependiente Colesterol Total pos administración del extracto acuoso de Proustia berberidifolia (tasa). UNJBG. TI. Efecto del extracto acuoso de Proustia berberidifolia (tasa) sobre los niveles de Colesterol Total plasmático en las ratas de experimentación. A grupos de 5 ratas hembras y 5 ratas machos se les administra el extracto en estudio. Los datos que se muestran en el panel A corresponden a los datos que resultan de comparar ambos grupos de machos, (control y tratamiento). Los datos que se muestran en el panel B corresponden a su similar pero con hembras. Todos los datos denotan similitud en el comportaniento de esta variable dependiente. 
Medias marginales estimadas de $\mathrm{HDL}$

En macho hembra $=$ macho

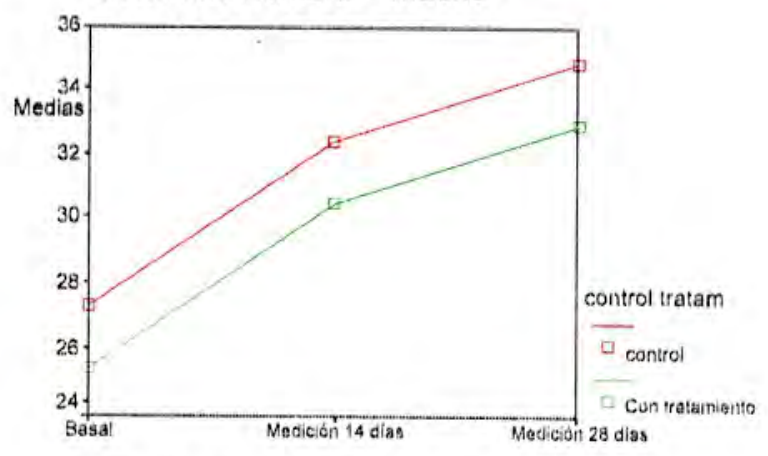

basal, $14 \mathrm{~d}, 28 \mathrm{~d}$
Medias marginales estimadas de HDL

En macho hembra $=$ hembra

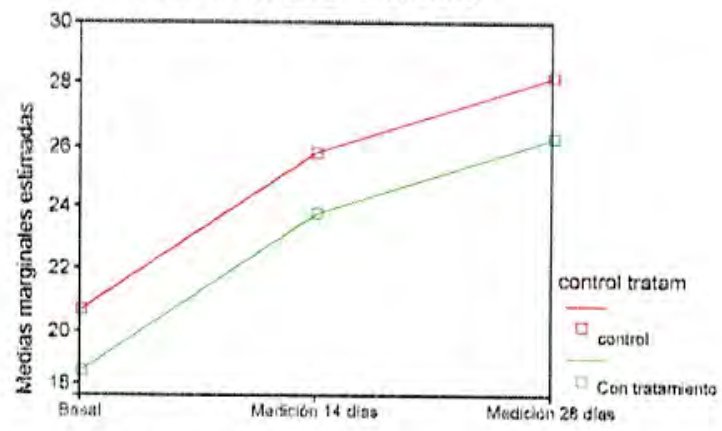

basal, 14d.28d

Figura $\mathrm{N}^{0}$ 2: Comportaniento de la variable dependiente hdl-colesterol pos administración del extracto acuoso de proustia berberidifolia.(tasa) UNJBG. TACNA. Ffecto del extracto acuoso de Proustia berberidifolia (tasa) sobre los niveles de HDL colesterol plasmático en las ratas de experimentación. A grupos de 5 ratas hembras y 5 ratas machos se les administra el extracto en estudio. Los datos que se muestran en el panel A corresponden a los datos que resultan de comparar ambos grupos de machos, (control y tratamiento). Los datos que se inuestran en el panel B corresponden a su similar pero con hembras. Todos los datos denotan similitud en el comportamiento de esta variable dependiente.

\section{Medias marginales estimadas de LDL}

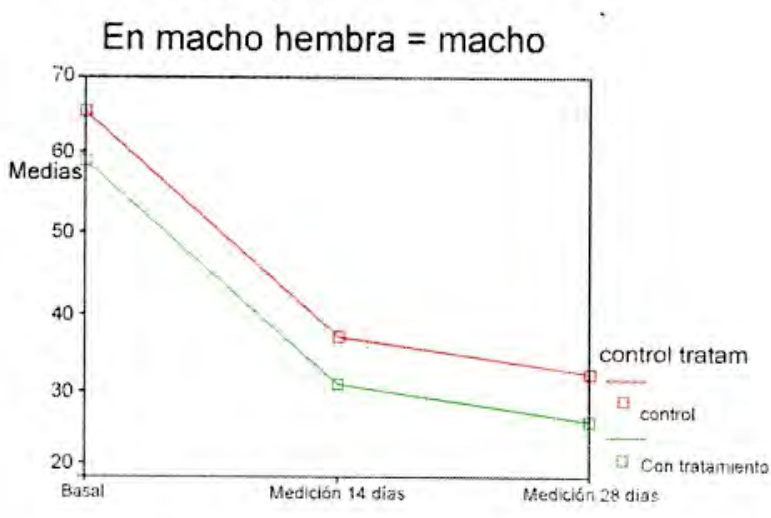

basal, $14 \mathrm{~d}, 28 \mathrm{~d}$

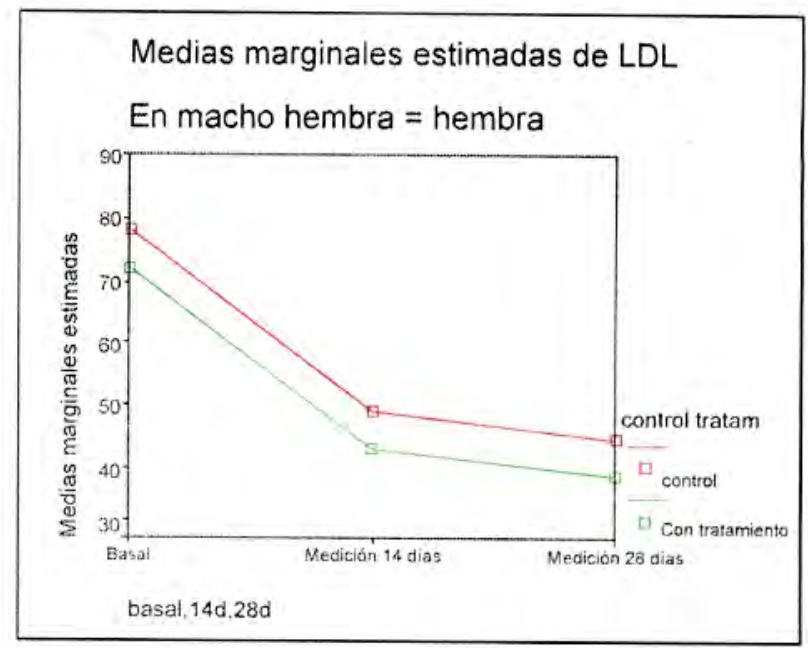

Figura $\mathrm{N}^{0}$ 3: Comportamiento de la variable dependiente Idl-colesterol pos administración del extracto acuoso de proustia berberidifolia.(Tasa) UNJBG. TACNA. Efecto del extracto acuoso de Proustia berberidifolia (tasa) sobre los niveles de LDL colesterol plasmático en las ratas de experimentación. A grupos de 5 ratas hembras y 5 ratas machos se les administra el extracto en estudio. Los datos que se muestran en el panel A corresponden a los datos que resultan de comparar ambos grupos de machos, (control y tratamiento). Los datos que se muestran en el panel B corresponden a su similar pero con hembras. Todos los datos denotan similitud en el comportamiento de esta variable dependiente.

niveles se mantienen dentro de los valores basales.

A nivel hepático los parámetros que reportan daño hepático y biliar son las transaminasas para el primer caso y las fosfatasas y las bilirrubinas para el segundo. Las transaminasas son enzimas intracelulares que están ubicadas en el citoplasma y/o mitocondrias y transfieren grupos amino de un aminoácido a un cetoácido. En estados normales aparecen en el plasma en bajisimas concentraciones, pero en ef caso de necrosis celular o alteración en la membrana plasmática pasan a la sangre en grandes cantidades, dependiendo de la extensión y profundidad del daño. De las dos enzimas (TGO, TGP) que determinamos la más especifica indicadora de lesión hepática es la transaminasa glutámicopiruvica
(TGP) inclusive se eleva antes de que aparezcan daño visible. Los resultados respecto a este tema reportan que el extracto utilizado no tiene efectos nocivos a nivel del hepatocito puesto que los niveles de ambas enzimas no se elevan, por el contrario, llama la atención los niveles disminuidos de forma significativa de TGP encontrados en sangre en los animales de experimentación, este aporte refleja el buen funcionamiento del higado a pesar del desgaste diario que sufre el higado y no a una incapacidad del hígado para producir estas enzimas por necrosis del hepatocito puesto que en esta patologia los niveles de TGO se elevan significativamente indicando daño masivo especialmente a nivel mitocondrial en los hepatocitos, suceso que no ocurre en nuestro caso, a lo que se adicionai las demás pruebas hepaticas que son 


\section{favorables}

Para el caso de daño extrahepático determinamos la concentración de fosfatasa alcalina aunque no es especifica para este tipo de órgano con ayuda de otros parámetros se puede inducir a un diagnostico. La medición del nivel de fosfatasas alcalinas es el mejor marcador de enfermedad colestásica hepática. Son enzimas no específicas del hígado y están elevadas durante el crecimiento. En el higado están ubicadas en la ocurre primariamente en desordenes colestásicos extra o intrahepáticos. La magnitud de la elevación no permite distinguir entre las diferentes patologías obstructivas y las infiltraciones malignas. De hecho mientras más elevada es la concentración dosada en sangre más probable es la existencia de colestasis extrahepática. Particularmente en nuestro estudio encontramos que los niveles de fosfatasa alcalina no presentan alteración a lo que se suma que los niveles de bilirrubina total y bilirrubina directa, en ambos casos, no están elevados: to que nos hace presunir que además de un buen funcionamiento hepático existe un buen drenaje a nivel de los conductos intra y extrahepáticos.

\section{CONCLUSIONES}

Del estudio se concluye que contradictoriamente las modificaciones observadas son positivas y están relacionados con efectos depurador, desintoxicador hepatoprotector e hipolipemiante.

\section{REFERENCIAS BIBLIOGRÁFICAS}

Schatzle M. Agathos M. Breit R. Allergic contact dermatitis from goldeurod (Herbasolidaginis) after systemic administration. Contact-Dermatitis. 1998 Nov; 39(5);271-2.

Bourgoignic JJ. et al."WWater: electrolytes, and acid-base balance in chromic renal failure". Semin Nephrol 1:2.91-111. 1981.

Carretero Colomer M. El regreso de las plantas medicinales. Hefame. 1998: (febrero): 75-80.

Chan TY.Interaction between warfarin and danshem (Salvia miltiorrhiza). Ann Pharmacother. $2000 \mathrm{Apr} ; 35$ (4): $501-4$.

Conz PA et al. Fucusvesiculosus: a nephrotoxic alga. Nephrol. Dial. Transplant. 1998 Fẹb: 13(2):526-7.

Duclos J, Goecke H. "Hierba del Clavo" (Geunthilvense) interfiere niveles de ciclosporina: potencial riesgo para trasplantados. Rev Med Chil. 2001 Jul: $129(7): 789-90$.

Foote J, Cohen B. Medicinal herb use and the renal patient. J. Rev. Nutr. 1998 Jan; $8(1): 40-2$

Frame $\mathrm{AD}$ et al. Plants from Puerto Rico with Anti Mycobacterium tuberculosis properties. Pr. Health Sei J.1998 Sep; $17(3): 243-52$.
Gaviln JC, Bermúdez FJ. Salgado F, Peña D. Fitoterapia y hepatitis. Rev Clínica Espanola. 1999: 199(10):693-4.

Izzo AA. Ernst E. Interaction between herbal medicines and prescribed drugs: a systematic review. Drugs 2001; 61 (15): 2163-75.

Krumme B, Endmeir R, Vanhaelen M. Walb D. Reversible Fanconisyn-drome after ingestion of a Chinese herbl "remedy"containing aristolochic acid. Nephrol. Dial. Transplant. 2001 Feb: 16(2): 400-2.

LOTT JA. WolfPL. (eds,):Clinical Enzimology: A C-Oriented Approach. Chicago. Year Book Medical Publishers 1986

Luper $\mathrm{S}$. A review of plantas used in the treatment of liver disease: part I. Altern Med Rev. 1998 Dec; 3 (6): 410-21.

Matthews MK Jr. Association of Gonkgobiloba with intracerebral hemorthage. Neurology. 1998 Jun;50 (6): 19334.

Myer S. Wohlmuth H. Echinacea-associated anaphylaxis. Med. H.Aust.1998 Jun 1: 168 (11):583-4

Oneschuk D, Bruerat E. The potential dangers of complementary therapy use in a patient with cancer. I Palliat Care. 1999Autumn: 15(3): 49-52.

Palop V. Cataln C. Rubio E., Martinez Mir I. Ginetomastia en un varóny ginseng. Med Clinica. 1999: 112(9): 758.

Parkman CA. Polypharmacy. herbal therapies, and elders.Case-Manager, 2001 Jul-Aug: 12 (4):32-4. (118)

Patsch W. Patsch JR, Gotto AM:“The hyperlipoproteinemias". Med Clin North Am73: 859-893.1989

Price SA. Wilson L.M. "Pathophysiology: Clinical Concepts of" disealse Processes". 2nded. New York. MeGraw-Hill. 1982

Rodriguez Lión ınl, Fernández del Pozo de Salaınanca mª del B. Zaragoza García F. Principios actisos de origen natural: flavonoides. Industria Farmacéutica. 1998: (septiembreoctubre): 87-92

Tomas ME et al Intensa colestasis asociada con hierbas medicinales. Rev Esp Enferm Fig. 1998 Jul; 90 (7):529-30.

Woo J. Treuting JJ. Cannon DC; Metabolic intermediates and inorganic ions, In Henry JB (ed) todd Sanford David sohn's Clinical Diagnosis and Management by Laboratory Methods. Philadelphia. WB Saunders. 1979 pp 259-304

\section{Correspondencia:}

Ricardo Ortiz Faucheux

Ciudad Universitaria - Fundo "Los Granados"

Av. Miraflores s/n. Tacna. Perú

Calle Arias Araguez $N^{\circ}$ 681. Tacna. Perú.

rof1962@hotmail.com 